\title{
Original
}

\section{Early Events Involving Glomerular Calcification Induced by Dibasic Sodium Phosphate Solution in Rats}

\author{
Noriko Tsuchiya ${ }^{1}$, Mikinori Torii ${ }^{1}$, Isao Narama $^{2}$, and Takane Matsui ${ }^{3}$ \\ ${ }^{1}$ Drug Safety Evaluation, Developmental Research Laboratories, Shionogi \& Co., Ltd., 3-1-1 Futaba-cho, Toyonaka, \\ Osaka 561-0825, Japan \\ 2 Department of Pathology, Faculty of Pharmaceutical Sciences, Setsunan University, Japan \\ ${ }^{3}$ Department of Pathological Science, Obihiro University of Agriculture and Veterinary Medicine, Japan
}

\begin{abstract}
To investigate the early changes involved in glomerular calcification, $360 \mathrm{mM} \mathrm{Na} \mathrm{HPO}_{4}$ was administered to rats at $8 \mathrm{~mL} / \mathrm{kg}$ once daily via the tail vein singly or repeatedly. Urinalysis was carried out on days $1,3,5$ and 8 of dosing, and rats were sacrificed on days 2, 4 and 9 for histopathological and electron microscopic examination of the kidneys. Following single dosing, there were no gross or histological findings, but electron microscopy revealed a number of vacuoles scattered within the Bowman's space. On day 4, minimal and focal mineralization was observed within the parietal epithelial cells. On day 9, mineralization was minimal to mild and localized within the parietal epithelial cells and glomerular basement membrane. Hypertrophy and increased mitotic figures were also frequently observed in the parietal epithelial cells. Low-density lamellar structures with effacement of podocytes, an increased number of microvilli and large amounts of debris filling the Bowman's space were the main electron microscopic changes on days 4 and 9 . Increased urinary protein excretion correlated well with the glomerular changes. Immunohistochemically, increased expression of desmin and decreased expression of podoplanin were evident in glomeruli on day 9. The numbers of PCNA-positive podocytes and parietal epithelial cells showed a tendency to increase on day 9. These results suggest that the onset of glomerular calcification is preceded by primary podocyte damage. (J Toxicol Pathol 2008; 21: 229-237)
\end{abstract}

Key words: rat, $\mathrm{Na}_{2} \mathrm{HPO}_{4}$, glomerulus, calcification, electron microscopy, immunohistochemistry

\section{Introduction}

We have previously investigated glomerular calcification in rats induced by intravenous bolus administration of 250 or $360 \mathrm{mM}$ dibasic sodium phosphate ( $\mathrm{Na}_{2} \mathrm{HPO}_{4}$, CAS number: 7558-79-4) solution for 14 days, which was used as a buffer vehicle, in rat subacute toxicity studies. Urinalysis revealed proteinuria on days 8 and 14, and calcium salt deposition in glomeruli occurred with a normal level of serum calcium on day 15. Electron microscopic examination of the kidney showed low-density lamellar structures in the Bowman's space, and peaks of phosphorus and calcium were detected by X-ray microanalysis in fine particles mixed with the lamellar structures ${ }^{1}$. Oral administration of $\mathrm{Na}_{2} \mathrm{HPO}_{4}$ at higher doses has been reported to cause calcium salt deposition within the renal tubular epithelium, and this is known as phosphate-

Received: 1 August 2008, Accepted: 9 September 2008 Mailing address: Noriko Tsuchiya, Drug Safety Evaluation, Developmental Research Laboratories, Shionogi \& Co., Ltd., 3-1-1 Futaba-cho, Toyonaka, Osaka 561-0825, Japan TEL: 81-6-6331-8081 FAX: 81-6-6332-6385

E-mail: noriko.tsuchiya@shionogi.co.jp induced nephropathy in dogs and rats ${ }^{2,3}$. However, there have been no reports of calcium salt deposition in glomeruli as a primary lesion in experimental studies or human cases with bolus $\mathrm{Na}_{2} \mathrm{HPO}_{4}$ injection.

The reason for calcium salt deposition chiefly in the glomerulus after bolus administration of highly concentrated $\mathrm{Na}_{2} \mathrm{HPO}_{4}$ solution is still unknown. The histopathological features of the glomerulus in our previous study suggest that deposition of calcium salt resulted from highly concentrated phosphorus and from dystrophic calcification secondary to cellular injury of podocytes or other components of the glomerulus. However, early changes indicating cellular injury were not evident because the kidneys were examined by light and electron microscopy at only one point after 14 days of administration.

To investigate the onset and early stages of the glomerular damage induced by $\mathrm{Na}_{2} \mathrm{HPO}_{4}$ solution, we examined the morphological and immunohistochemical changes in the kidneys and conducted urinalysis on days 2, 4 and 9. In the present study, we identified early events in glomeruli, and herein, we describe the possible pathogenesis of glomerular calcification. 


\section{Materials and Methods}

\section{Chemicals}

$\mathrm{Na}_{2} \mathrm{HPO}_{4} \cdot 12 \mathrm{H}_{2} \mathrm{O}$ (Kanto Chemical Co., Inc. Tokyo, Japan) was diluted with physiological saline to produce 360 $\mathrm{mM}$ solutions $\left(409.6 \mathrm{mg} / \mathrm{kg} /\right.$ day $\left.\mathrm{Na}_{2} \mathrm{HPO}_{4}\right)$. On the basis of our previous study ${ }^{1}$, we selected $360 \mathrm{mM}$ solution to induce stable glomerular calcification. The $\mathrm{Na}_{2} \mathrm{HPO}_{4}$ solution was adjusted to $\mathrm{pH} 5.5-6.5$ using $1 \mathrm{~N}$ sulfuric acid, and its osmolarity was $748 \mathrm{mOsm} / \mathrm{kg}$.

\section{Animals and housing conditions}

All experimental procedures were conducted after approval of the study by the Institutional Animal Care and Use Committee of Shionogi Research Laboratories. Twenty-four male, Sprague-Dawley rats (Jcl:SD) were obtained at 5 weeks of age from Clea Japan Inc. (Shiga, Japan) and acclimated for a week before treatment. Fifteen rats, randomly allocated to 3 groups of 5 animals each, received single or repeated administrations of $\mathrm{Na}_{2} \mathrm{HPO}_{4}$ solution. The remaining 9 rats were used as controls. The rats were housed individually in plastic cages in an animal room kept under controlled conditions (temperature of $23 \pm$ $3^{\circ} \mathrm{C}$, humidity of $50 \pm 20 \%$, lighting for $12 \mathrm{hrs}$ between 8:00 A.M. and 8:00 P.M., ventilation 10 times per hour). The rats were fed pelleted diet (CA-1, Clea Japan Inc., Tokyo) and provided water ad libitum via an automatic watering system after filtration through 30 - and $3-\mu \mathrm{m}$-pore filters followed by UV irradiation.

\section{Experimental design (Fig. 1)}

The rats were administered $360 \mathrm{mM} \mathrm{Na} \mathrm{HPO}_{4}$ at $4 \mathrm{~mL} /$ $\mathrm{kg} / \mathrm{min}$ for 2 minutes once daily via the tail vein through a polyethylene catheter connected to a plastic syringe mounted on an infusion pump (STC-525, Terumo Corp., Tokyo). The control animals received physiological saline in the same way. Clinical signs and body weights were observed and measured each day. Five treated and three control rats were sacrificed on days 2, 4 and 9.

\section{Urinalysis}

All rats were kept in individual metabolic cages and deprived of food and water, and urine was collected 4 hours after dosing on days $1,3,5$ and 8 . Protein, color, $\mathrm{pH}$, glucose, ketone bodies, urobilinogen, bilirubin and occult blood were measured with Clinitek 200 Plus (Bayer Corporation Diagnostics Division, Elkhart, IN, USA) for qualitative analysis. Urinary excretion of albumin is detected as proteinuria, and the measurement range was as follows: 0 was negative, $15 \mathrm{mg} / \mathrm{dl}$ was, $\pm 30 \mathrm{mg} / \mathrm{dl} \mathrm{was}+$, $100 \mathrm{mg} / \mathrm{dl}$ was $2+, 300 \mathrm{mg} / \mathrm{dl}$ was $3+$ and $1000 \mathrm{mg} / \mathrm{dl}$ was $4+$.

\section{Histopathology}

Rats were euthanized by exsanguination by cutting both the abdominal aorta and vena cava under pentobarbital sodium anesthesia and were then necropsied. The kidneys

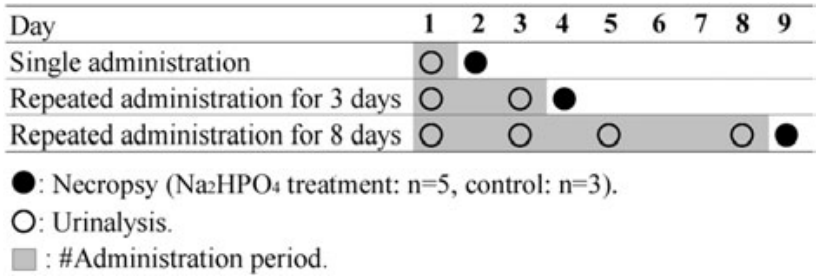

Fig. 1. Experimental protocol, showing time course of dosing of dibasic sodium phosphate and saline for the rats.

were removed and weighed together (Mettler PM400). The following organs were microscopically examined: kidneys, liver, lung, heart, spleen, urinary bladder, brain, thyroid, parathyroid, pituitary, adrenal glands, testes, spinal cord, stomach, small intestine, large intestine and femur (including bone marrow). These organs were fixed in 10\% neutral buffered formalin, processed routinely and embedded in paraffin. Paraffin sections were prepared and stained with hematoxylin and eosin (H\&E). The kidney sections were also stained with von Kossa and periodic acidSchiff (PAS) reagents and periodic acid-methenamine-silver (PAM) stain.

\section{Immunohistochemistry}

To assess the proliferating activity and functional changes of the glomerulus, kidney sections were subjected to indirect immunoperoxidase staining using the following primary antibodies: PCNA (proliferating cell nuclear antigen, 1:200, Dako Corporation, Carpinteria, CA, USA), podoplanin (specific markers for podocytes and parietal epithelial cells, 1:1600, P1995, Sigma, USA) and desmin (1:100, M0760, Dako Corporation). The kidney sections were deparaffinized, rehydrated and then incubated with pepsin (Dako Corporation) at $37^{\circ} \mathrm{C}$ for 20 min or boiled in citric acid (10 mmol; $\mathrm{pH} 6.0$ ) for 10 minutes to unmask antigens. The sections were immersed in PBS ( $\mathrm{pH} 7.4$ ) containing $3 \% \mathrm{H}_{2} \mathrm{O}_{2}$ to inactivate endogenous peroxidase. Equilibration buffer was applied to the sections for $30 \mathrm{~min}$ at room temperature. Immunoperoxidase staining was performed in accordance with the Nichirei-Histofine Simple stain kit (Nichirei Biosciences Inc.) or Vectastain ABC kit (Vector Laboratories, Burlingame, CA, USA), and the staining was visualized with diaminobenzidine. They were then counter stained with Mayer's hematoxylin, dehydrated and mounted.

Semiquantitative analysis was performed for the results of immunohistochemical examination by assessing the amount of podoplanin or desmin expression in glomeruli and counting the number of PCNA-positive cells per field at $\times 400$ magnification. Fifty to 60 glomeruli in 2 sections were randomly chosen and examined $(n=3$ per group at each time point). The numbers of PCNA-positive cells per glomerulus were counted ${ }^{4}$. The extent of glomerular expression of podoplanin was also evaluated. Scores were assigned to individual glomeruli in each section as follows: 0 , no expression; 1, expression in few glomeruli; 2, up to $50 \%$ of 
Table 1. Detection of Proteinuria by Urinalysis

\begin{tabular}{|c|c|c|c|c|c|c|c|c|}
\hline \multirow[t]{2}{*}{ Treatment } & \multicolumn{2}{|c|}{ Day 1} & \multicolumn{2}{|c|}{ Day 3} & \multicolumn{2}{|c|}{ Day 5} & \multicolumn{2}{|c|}{ Day 8} \\
\hline & Control & $\mathrm{Na}_{2} \mathrm{HPO}_{4}$ & Control & $\mathrm{Na}_{2} \mathrm{HPO}_{4}$ & Control & $\mathrm{Na}_{2} \mathrm{HPO}_{4}$ & Control & $\mathrm{Na}_{2} \mathrm{HPO}_{4}$ \\
\hline Number of animals & 9 & 15 & 6 & 10 & 3 & 5 & 3 & 5 \\
\hline Negative & $0^{\#}$ & 0 & 0 & 0 & 0 & 0 & 0 & 0 \\
\hline Minimal* & 4 & 11 & 6 & 4 & 2 & 0 & 3 & 0 \\
\hline Mild & 5 & 4 & 0 & 4 & 1 & 1 & 0 & 0 \\
\hline Moderate & 0 & 0 & 0 & 2 & 0 & 3 & 0 & 1 \\
\hline Marked & 0 & 0 & 0 & 0 & 0 & 1 & 0 & 4 \\
\hline
\end{tabular}

*Minimal ( $($ ): $<15$ mg/dl; Mild (+): 30 mg/dl; Moderate (2+): 100 mg/dl; Marked (3+ to 4+): > $300 \mathrm{mg} / \mathrm{dl}$.

"Number of animals affected.

glomeruli with positive cells; $3,50-75 \%$ of glomeruli with positive cells; 4, positive cells all over the glomeruli. For analysis of desmin immunohistochemistry, the capillary tuft was divided into 4 quarters, and the following scoring system was used: 0 , no expression; 1 , desmin-positive cells in $25 \%$ of the capillary tuft; 2 , desmin-positive cells in $50 \%$ of the capillary tuft; 3 , desmin-positive cells in $75 \%$ of the capillary tuft; and 4 , desmin-positive cells in $100 \%$ of the capillary tuft. The final score per section was then calculated as the weighted mean as follows:

$\mathrm{S}_{\text {desmin }}=\left[\left(0 \times \mathrm{N}_{0}\right)+\left(1 \times \mathrm{N}_{1}\right)+\left(2 \times \mathrm{N}_{2}\right)+\left(3 \times \mathrm{N}_{3}\right)+\left(4 \times \mathrm{N}_{4}\right)\right] /\left[\mathrm{N}_{0}\right.$ $\left.+\mathrm{N}_{1}+\mathrm{N}_{2}+\mathrm{N}_{3}+\mathrm{N}_{4}\right]$,

where $\mathrm{Ni}(i=0$ to 4$)$ is the number of glomeruli in each category ${ }^{4,5}$.

\section{Transmission electron microscopy (TEM)}

For electron microscopic observation, kidneys that were excised from 2 animals per group at each time point were fixed in $3 \%$ glutaraldehyde and $2 \%$ osmic acid and embedded in epoxy resin. Ultrathin sections were stained with uranyl acetate and lead citrate and examined under an electron microscope (JEM-1010, JEOL Ltd., Tokyo).

\section{Statistical analysis}

Statistical significance was analyzed by Student's t-test for body and kidney weights and the Mann-Whitney test for immunohistochemistry. All data are expressed as means \pm S.D.

\section{Results}

\section{Clinical signs and urinalysis}

Neither abnormal clinical signs nor body weight changes were observed in any group.

No increase in urinary protein was detected in the treated rats on day 1 in comparison with the control rats. Increased urinary protein excretion was first detected on day 3 in 2 out of 10 treated rats; the values in these animals were $100 \mathrm{mg} / \mathrm{dl} / 4 \mathrm{hr}$ (the dipsticks showed 2+). Almost all treated animals had an increased urinary protein excretion rate on day 5 , with values ranging from 100 to $1000 \mathrm{mg} / \mathrm{dl} / 4 \mathrm{hr}(2+$ to $4+$ ). By day 8 , all treated rats showed moderate to severe proteinuria. In the control group, although several rats had minimal to mild proteinuria, no time course increase was observed (Table 1). No other abnormalities were observed.

\section{Kidney weights and gross examination}

There were no statistically significant differences in the kidney weights of the phosphate-treated rats in comparison with the control group.

\section{Histopathology}

No significant changes were observed in the saline control animals. There were no lesions in any of the glomeruli of the five animals after single administration of $360 \mathrm{mM} \mathrm{Na} \mathrm{HPO}_{4}$. On day 4 of treatment, minimal and focal deposition of fine basophilic granules was detected within the parietal epithelial cells in 2 animals, but there were no remarkable lesions in the capillary endothelium or glomerular basement membrane (GBM). Basophilic granules were confirmed to be calcium depositions by von Kossa staining (Fig. 2A).

After 8-day repeated dosing, focal calcification of the GBM and mesangium was evident along with minimal to mild deposition of calcium salts within the parietal epithelial cells. Hypertrophy and increased mitotic figures were also accompanied by calcification in the parietal epithelial cells (Fig. 2B).

There were no lesions observed in any other organs including the bone and parathyroid.

\section{Immunohistochemistry}

There were no differences in podoplanin expression or the number of PCNA-positive cells between the treated and control animals on days 2 and 4 because almost all glomeruli showed strong expression of podoplanin but contained few PCNA-positive cells. The numbers of PCNA-positive nuclei in podocytes and parietal epithelial cells tended to increase on day 9 (Fig. 3A, 4).

In the control rats, podoplanin was expressed in a linear pattern on the cell membranes of podocytes and parietal epithelial cells of the Bowman's capsule. The number of podoplanin-positive podocytes and parietal epithelial cells tended to be lower on days 4 and 9, and the differences were significant for the podocytes and parietal epithelial cells on day 9 (Fig. 3B, 4). 

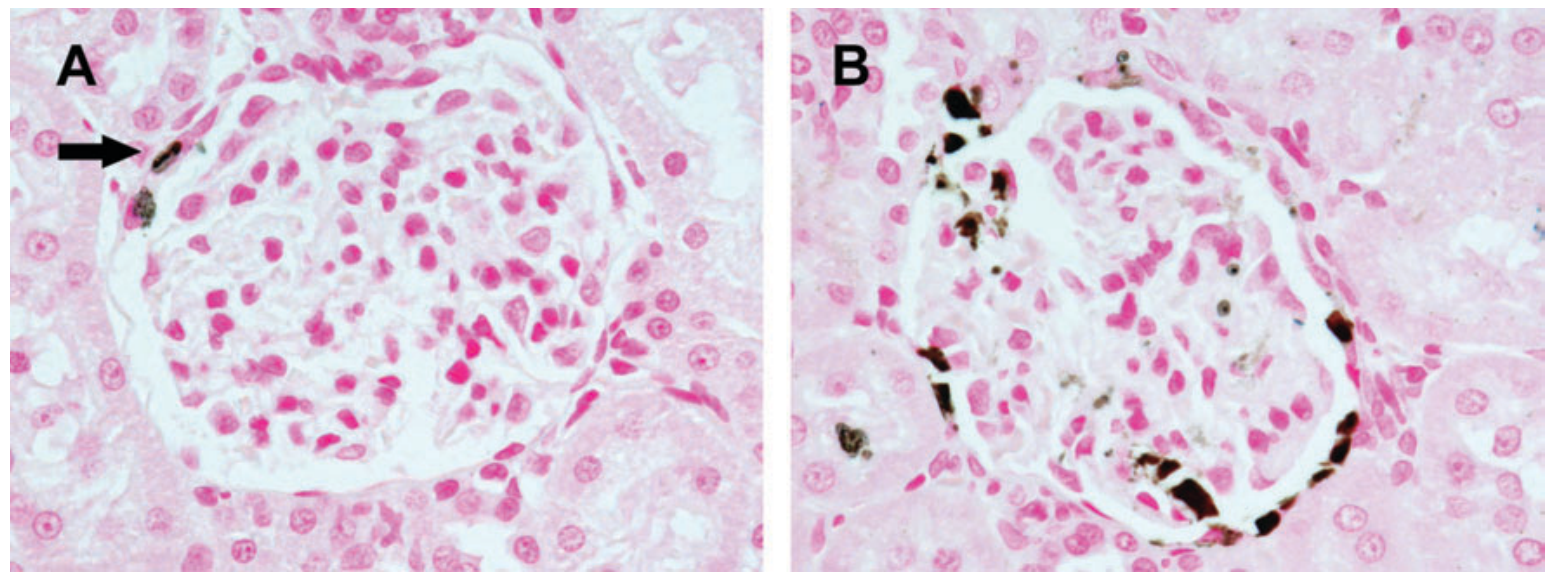

Fig. 2. Glomerular calcification in phosphate-treated rats. (A) Slight calcium deposition on the parietal epithelial cells on day 4 (arrow); (B) Calcium deposition on the parietal epithelial cells of the Bowman's capsule and podocytes on day 9. von Kossa stain.
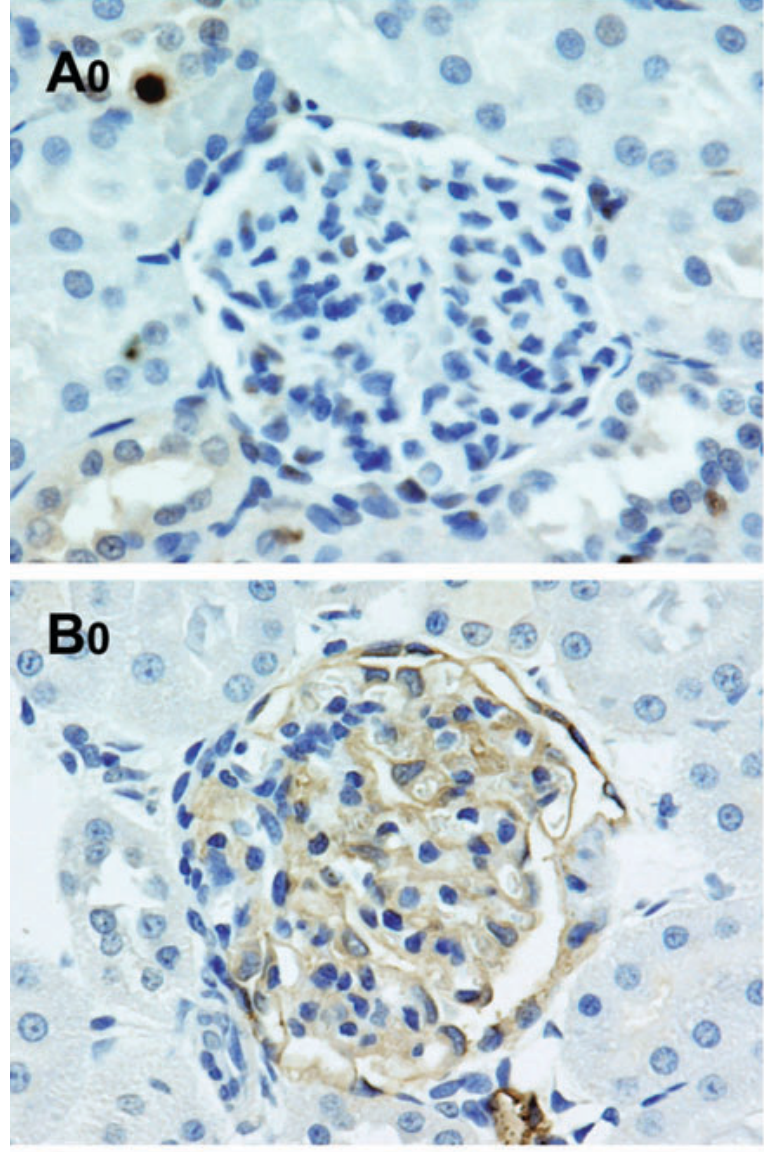

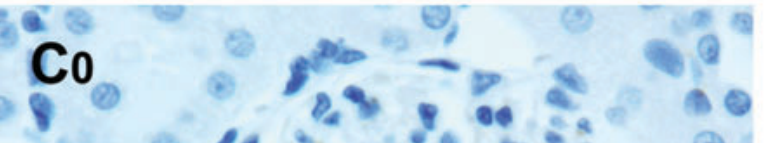

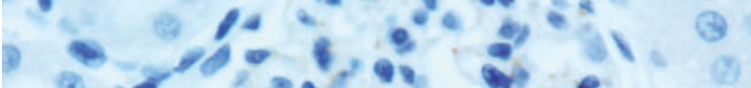

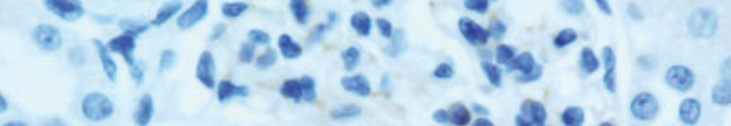
$000^{\circ},-0.260^{\circ}$

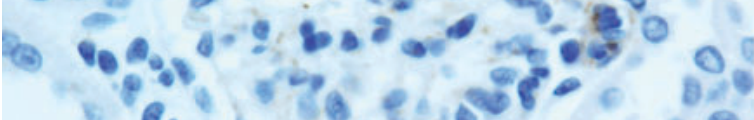
\&.

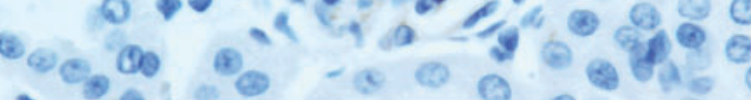
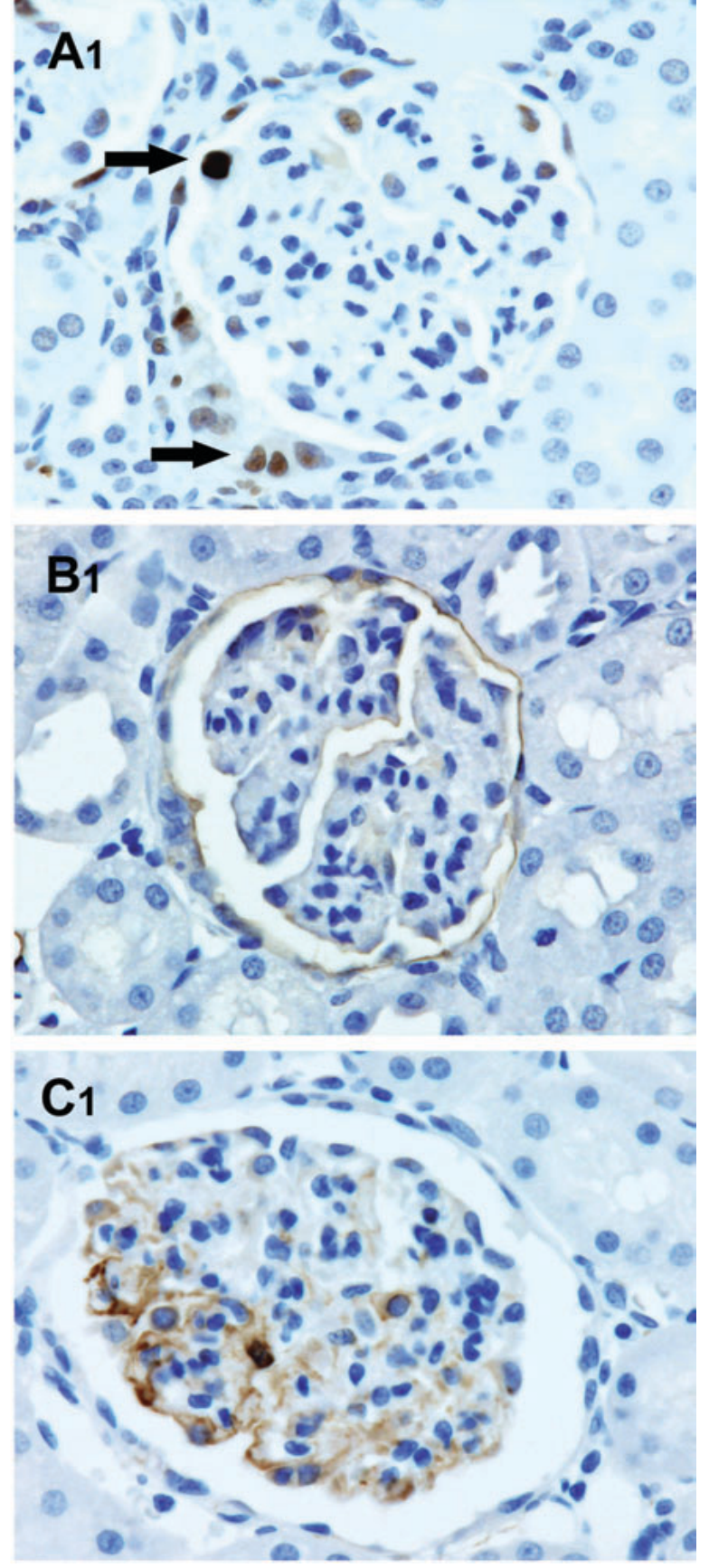
Conversely, anti-desmin antibody mainly reacted with the mesangial and vascular smooth muscle cells, and very weak staining was observed in the podocytes in the control kidney. Desmin expression expanded over $25 \%$ of the glomerular tuft on days 4 and 9 and was significantly higher than in the controls on day 9 (Fig. 3C, 4).

\section{Electron microscopy}

Following single administration of $360 \mathrm{mM} \mathrm{Na} \mathrm{HPO}_{4}$ solution, a number of small vacuoles with unit membranes
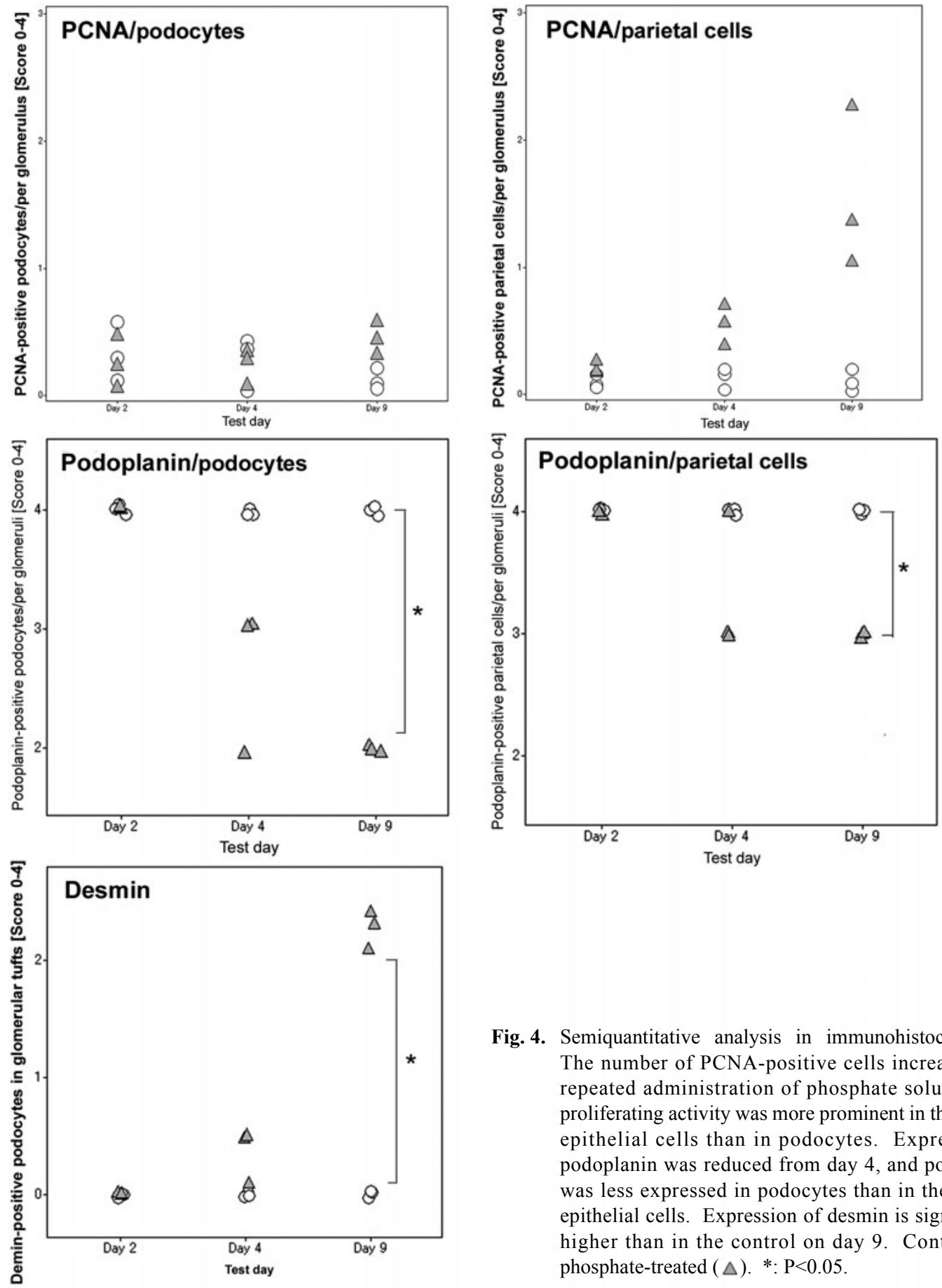

Fig. 4. Semiquantitative analysis in immunohistochemistry. The number of PCNA-positive cells increased with repeated administration of phosphate solution, and proliferating activity was more prominent in the parietal epithelial cells than in podocytes. Expression of podoplanin was reduced from day 4 , and podoplanin was less expressed in podocytes than in the parietal epithelial cells. Expression of desmin is significantly higher than in the control on day 9. Control $(O)$, phosphate-treated $(\triangle)$. *: $\mathrm{P}<0.05$.

Fig. 3. Immunohistochemistry for proliferation cell nuclear antigen (PCNA), podoplanin and desmin. None of the glomerular cells expressed PCNA in the control glomerulus (A0), whereas there was positive PCNA expression in several podocytes and the parietal epithelial cells on day 9 (A1, arrow). Podoplanin was found in a linear pattern on the cell membranes of podocytes and the parietal epithelial cells of the control glomerulus (B0). Podoplanin expression was reduced in both podocytes and the parietal epithelial cells on day 9, but the podoplanin-positive podocytes were greatly diminished (B1). Expression of desmin was rare in the control glomerulus (C0). Desmin was present in some tufts on day $9(\mathrm{C} 1)$. 
were scattered within the Bowman's space (Fig. 5A, 5B). The membranes of the vacuoles were between 20 and $50 \mathrm{~nm}$ in thickness. High-density particles adhered to the membranes and mixed with clusters of debris within the Bowman's space. A small number of vacuoles were also seen in the cytoplasm of the podocytes, and some of the small vacuoles floating within the Bowman's space seemed to show contiguity with the cytoplasmic vacuole of the podocyte. The capillary endothelium showed a partially higher electron density and irregular thickening (Fig. 5C).

On day 4, low-density lamellar structures were observed within the Bowman's space, the GBM and the mesangial matrix. The lamellar structures in the Bowman's space contained small particles and were larger in size than those in the GBM and mesangial matrix. The lamina densa of the GBM was partially obscure due to deposition of lamellar structures. Some of these lamellar structures within the GBM protruded into the vascular cavity, and the podocytes lining the GBM exhibited hypertrophy. Furthermore, the cell processes of the podocytes often became fused with each other, the number of microvilli increased, and the Bowman's space was filled with large amounts of debris.

On day 9, degeneration of the podocytes was more severe than on day 4 , and accreted lamellar structures accumulated partially within parietal epithelia, podocytes and GBM. Large amounts of lamellar structures were deposited at each site as a result of hypertrophy and swelling (Fig. 6).

The results are shown in Table 2.

\section{Discussion}

The present study shows the early glomerular changes in male rats administered dibasic sodium phosphate solution for 8 days and sacrificed on days 2, 4 and 9. On day 9, calcification was observed in all parts of glomeruli, and this lesion was comparable with that found in our previous study when rats were administered dibasic sodium phosphate solution for 14 days. Electron microscopic examination afforded abundant evidence concerning the onset of degeneration of the parietal epithelial cells and podocytes.

Increased urinary protein excretion was first detected on day 3, and all phosphate-treated rats showed proteinuria by day 8 . The glomerular changes were consistent with the onset of marked proteinuria, which was considered to be due to protein leakage secondary to glomerular injury characterized by degeneration of podocytes. Polycationic agents induce charge reduction in the glomerular filtration barrier followed by functional evidence of injury, which manifests as proteinuria ${ }^{6}$. Thus, proteinuria appears to be a significant biomarker of podocyte injury due to glomerular calcification.

Puromycin aminonucleoside (PAN) has been found to be a direct cytotoxic model that injures podocytes, causing underproduction of sialoglycoprotein and proteoglycan by them. The glomeruli of PAN-treated Sprague-Dawley rats

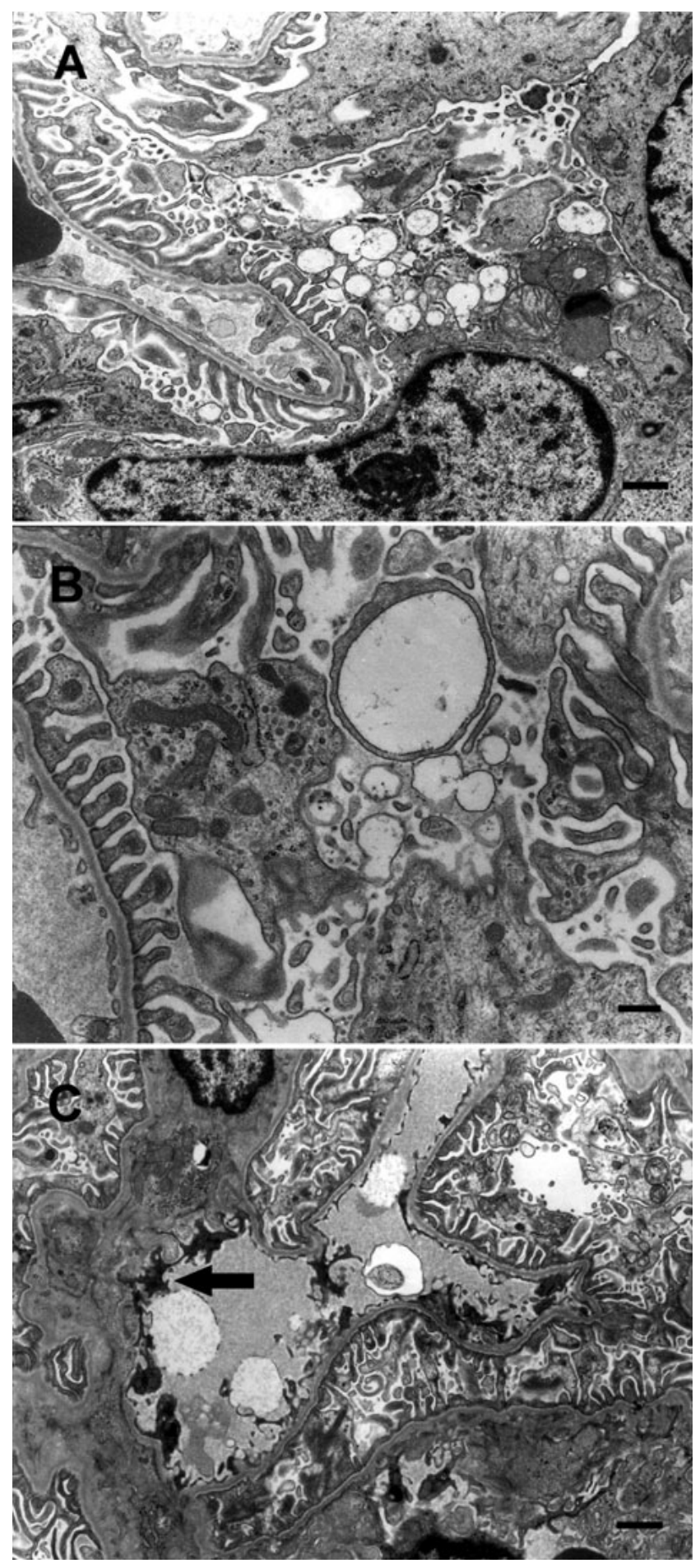

Fig. 5. Glomerular ultrastructure in a rat that received a single phosphate dosing. (A) A number of small vacuoles with unit membranes are scattered within the Bowman's space. Scale bar $=1 \mu \mathrm{m}$. (B) Vacuolation within the podocyte. Scale bar $=500 \mathrm{~nm}$. (C) The capillary endothelium is partially dark in color (arrow) and shows irregular thickening. Scale bar $=2$ $\mu \mathrm{m}$.

after treatment for 4 days showed extensive replacement of podocytic foot processes by broad expanses of epithelial cytoplasm. The epithelium also showed a small number of 
Table 2. Findings for the Dibasic Sodium Phosphate Solution Treated Rats

\begin{tabular}{|c|c|c|c|c|}
\hline \multirow{2}{*}{ Findings } & & \multicolumn{3}{|c|}{ Days of administration } \\
\hline & & Single & 3 days & 8 days \\
\hline Urinalysis: proteinuria & & - & $\mathrm{P}$ & $\mathrm{P}$ \\
\hline \multirow[t]{8}{*}{ Microscopy } & Calcification: parietal epithelial cells & - & $\mathrm{P}$ & $\mathrm{P}$ \\
\hline & GBM/podocytes & - & - & $\mathrm{P}$ \\
\hline & mesangium & - & - & $\mathrm{P}$ \\
\hline & Hypertrophy of parietal epithelial cells & - & - & $\mathrm{P}$ \\
\hline & Proliferation and/or activation of parietal epithelial cells & $\mathrm{P}$ & $\mathrm{P}$ & $\mathrm{P}$ \\
\hline & Decrease of podoplanin expression in parietal epithelial cells & - & - & $\mathrm{P}$ \\
\hline & Decrease of podoplanin expression in podocytes & - & $\mathrm{P}$ & $\mathrm{P}$ \\
\hline & Desmin expression in glomeruli & - & - & $P$ \\
\hline \multirow[t]{6}{*}{ Electron microscopy } & Small vacuoles in Bowman's space and podocytes & $P$ & - & - \\
\hline & Lamellar body deposition & - & $\mathrm{P}$ & $\mathrm{P}$ \\
\hline & Effacement/degeneration of podocytes & - & $\mathrm{P}$ & $\mathrm{P}$ \\
\hline & Increase of microvilli & - & $\mathrm{P}$ & $\mathrm{P}$ \\
\hline & Thickening of GBM & - & - & $\mathrm{P}$ \\
\hline & Electron-dense capillary endothelium & $\mathrm{P}$ & $\mathrm{P}$ & $P$ \\
\hline
\end{tabular}

P: present; -: not present.

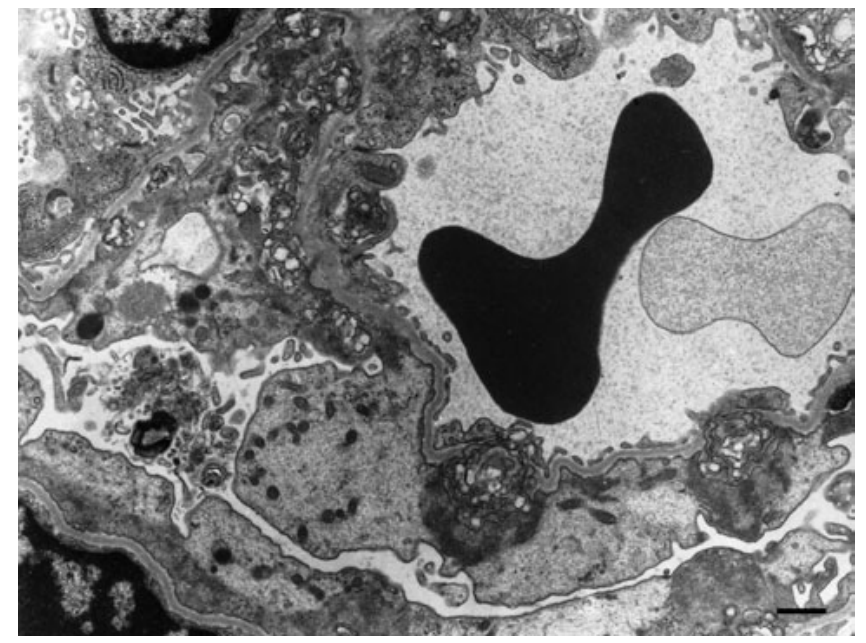

Fig. 6. Glomerular ultrastructure in an 8-day phosphate-treated rat. The large depositions of lamellar structures in the parietal epithelial cells, podocytes and the glomerular basement membrane seem to cause hypertrophy and swelling. Scale bar $=1 \mu \mathrm{m}$.

cytoplasmic vacuoles, which contained homogenous finely granular material ${ }^{7}$. In the present study, TEM revealed vacuoles smaller in size than those of the PAN model, and most of them were floating in the Bowman's space. There was no clear morphological evidence of damaged podocytes; however, they might be distal parts of intracytoplasmic vacuoles of the podocytes or parts of the podocytes that had degenerated and become detached from the GBM. Some small vacuoles in the Bowman's space seemed to continue into the cytoplasm of the podocyte. Podocytes may be affected by sodium phosphate solution and become vacuolated, but no specific lesion occurred, and this is similar to the case with PAN.
On day 4, small lamellar structures appeared within the Bowman's space, parietal epithelial cells, podocytes, GBM and mesangial matrix in addition to the vacuoles. Vacuoles were very likely to have been present in the lamellar structure on day 4 because vacuoles and lamellar structure also appeared in the Bowman's space. Deposition of phosphorus and calcium in fine particles mixed with lamellar structures has been confirmed by X-ray microanalysis in a previous study ${ }^{1}$. The glomerulus has a complex structure, the stability of which depends on cooperation among mesangial cells, podocytes and the basement membrane formed between these cells. One important question that arises concerns why lamellar structures were observed within the various glomerular sites with almost the same time course, regardless of the great variety of glomerular cells according to function.

In the present study, the PCNA-positive podocytes and parietal epithelial cells showed a tendency to increase on day 9. The parietal epithelial cells are not terminally differentiated like podocytes and therefore are capable of proliferation during glomerular injury ${ }^{8}$. Our data suggests that regenerative proliferation of the parietal epithelial cells of the Bowman's capsule occurred in response to injury induced by the phosphate solution. In the kidneys of rats 1 day after birth, podocytes in maturing glomeruli are commonly labeled in PCNA. However, PCNA staining is absent in the kidney of the adult rat ${ }^{9}$. The normal podocyte in a matured glomerulus is a terminally differentiated cell that relinquishes mitotic capability ${ }^{8}$. Nuclear division without cell division does on occasion occur, resulting in binucleated and multinucleated podocytes, an event that is most marked in nephropathic cystinosis ${ }^{10}$. Cell division would demand reorganization of the cell cytoskeleton; this would disrupt the foot processes, affecting attachment to the GBM and filtration selectivity of the slit diaphragm ${ }^{8}$. 
Although podocytes have previously been regarded as fixed in a terminally differentiated state, it is now clear that reentry into the cell cycle occurs in a few glomerular diseases, such as HIV nephropathy ${ }^{10}$, other collapsing forms of focal segmental glomerulosclerosis ${ }^{11}$ and PAN nephropathy ${ }^{12-14}$, leading to the concept of dysregulated podocytes. The tendency of PCNA-positive podocytes to increase, observed in the present study, suggests an association with their attempt to enter mitosis or cell division.

In the kidneys of adult rats, expression of podoplanin shows an exclusively linear pattern on the cell membranes of podocytes and the parietal epithelial cells of the Bowman's capsule $^{15}$. The decreased numbers of podoplanin-positive podocytes in the glomeruli of the phosphate-treated rats on day 9 suggests impairment of podocyte function due to administration of the $\mathrm{Na}_{2} \mathrm{HPO}_{4}$ solution.

In the control kidney, anti-desmin antibody mainly reacted with the mesangial and vascular muscle cells, and very weak staining was observed in podocytes. Desmin staining in the glomeruli of the phosphate-treated rats was observed in podocytes, and the staining expanded over $25 \%$ of the glomerular tuft. In PAN-induced nephrosis, conspicuously enhanced staining with desmin was seen in podocytes on day 10 , whereas no change in desmin staining was detected in the mesangium. Upregulation of intermediate filament proteins may play a critical role in the morphological changes of podocytes in response to injury ${ }^{16}$.

The true pathogenesis of phosphate-induced glomerular calcification in rats remains unclear. Deposition of calcium salt may result from dystrophic calcification secondary to the cellular injury of the glomerulus and from highly concentrated phosphorus. We speculate that the pathogenesis of the glomerular changes in the early stages and the process of lamellar structure formation occurred in several areas as follows. First, podocytes were directly impaired by a single injection of $\mathrm{Na}_{2} \mathrm{HPO}_{4}$ solution and exhibited a small number of cytoplasmic vacuoles. Some of the injured podocytes became detached from the GBM and entered the Bowman's space, after which lamellar structures may have been formed with altered podocytes. Before the impaired podocytes became completely detached from the GBM, the GBM may have been repaired by the remaining podocytes, with the renewed GBM covering the former GBM. Thus, the lamellar structure appeared to be stranded in a deeper part of the GBM. A second possibility is that the $\mathrm{Na}_{2} \mathrm{HPO}_{4}$ solution directly affected the GBM and mesangium, which include the common elements of type IV collagen and polyanionic proteoglycans, particularly heparan sulfate. Proteinuria arose as a result of charge reduction in the glomerular filtration barrier followed by functional evidence of injury with a polycationic agent such as protamine ${ }^{6}$. If $\mathrm{PO}_{4}{ }^{2-}$ injures the $\mathrm{GBM}$ and mesangium, as polycationic agents do, there is a high possibility of it causing the same morphologic changes. A third possibility is that, as the glomerular endothelium was partially dark in color and showed irregular thickening in the present study, it is likely that the capillary endothelium is directly damaged and degenerated by the $\mathrm{Na}_{2} \mathrm{HPO}_{4}$ solution. Glomerular endothelial injuries inevitably affect mesangial and epithelial cells and presumably modify the progression of renal disease by reciprocally interacting with them ${ }^{17}$.

These results show that phosphate-induced glomerular lesions occur with repeated administration of $\mathrm{Na}_{2} \mathrm{HPO}_{4}$ solution for 3 days. Few morphological changes, which were observable only by electron microscopy, may be important as factors preceding glomerular calcification. Since the glomerular changes closely paralleled the onset of marked proteinuria, urinalysis is desirable during administration. Further treatment conducted over longer periods could lead to more severe glomerular degeneration and subsequent tubular degeneration.

Acknowledgements: We are grateful to Takako Miyoshi, Mariko Hirano, Kae Fujisawa and Koichi Masuno for their technical assistance. We also thank our many colleagues for their support during our work.

\section{References}

1. Tsuchiya N, Matsushima S, Takasu N, Kyokawa Y, and Torii M. Glomerular calcification induced by bolus injection with dibasic sodium phosphate solution in Sprague-Dawley rats. Toxicol Pathol. 32: 408-412. 2004.

2. Scheneider P, Ober KM, and Ueberberg H. Contribution to the phosphate-induced nephropathy in the dog. Comparative light and electron microscopic investigations of the proximal tubule after oral application of $\mathrm{K}_{2} \mathrm{HPO}_{4}, \mathrm{Na}_{2} \mathrm{HPO}_{4}, \mathrm{KCl}$ and NaCl. Exp Pathol. 19: 53-65. 1981.

3. Matsuzaki H, Kikuchi T, Kajita Y, Masuyama R, Uehara M, and Goto S. Comparison of various phosphate salts as the dietary phosphorus source on nephrocalcinosis and kidney function in rats. J Nutr Sci Vitaminol. 45: 595-608. 1999.

4. Gross ML, Ritz E, Schoof A, Adamczak M, Koch A, Tulp O, Parkman A, El-Shakmak A, Szabo A, and Amann K. Comparison of renal morphology in the streptozotocin and the SHR/N-cp models of diabetes. Lab Invest. 84: 452-464. 2004.

5. Macconi D, Bonomelli M, Benigni A, Plati T, Sangalli F, Longaretti L, Conti S, Kawachi H, Hill P, Remuzzi G, and Remuzzi A. Pathophysiologic implications of reduced podocyte number in a rat model of progressive glomerular injury. Am J Pathol. 168: 42-54. 2006.

6. Khan KNM and Alden CL. Kidney. Handbook of Toxicologic Pathology. WM Haschek, CG Rousseaux, MA Wallig (eds). Academic Press, San Diego, California. 259315. 2002.

7. Messina A, Davies DJ, Dillane PC, and Ryan GB. Glomerular epithelial abnormalities associated with the onset of proteinuria in aminonucleoside nephrosis. Am J Pathol. 126: 220-229. 1987.

8. Bonsib SM. Renal anatomy and histology. In: Heptinstall's Pathology of the Kidney. JC Jennette, JL Olson, MM Schwartz, FG Siva (eds). Lippincott Williams \& Wilkins, Philadelphia. 26-41. 2007.

9. Omori S, Hida M, Ishikura K, Kuramochi S, and Awazu M. Expression of mitogen-activated protein kinase family in rat renal development. Kidney Int. 58: 27-37. 2000. 
10. Barisoni L, Kriz W, Mundel P, and D'Agati V. The dysregulated podocyte phenotype: a novel concept in the pathogenesis of collapsing idiopathic focal segmental glomerulosclerosis and HIV-associated nephropathy. J Am Soc Nephrol. 10: 51-61. 1999.

11. Bonsib SM and Horvath F Jr. Multinucleated podocytes in a child with nephrotic syndrome and Fanconi's syndrome: A unique clue to the diagnosis. Am J Kidney Dis. 34: 966-971. 1999.

12. Yamazaki T. Podocytic degeneration and regeneration in puromycin aminonucleoside nephropathy in the rat. Pahol Int. 45: 465-472. 1995.

13. Sasaki T, Jyo Y, Tanda N, Kawakami Y, Nohno T, Tamai H, and Osawa G. Changes in glomerular epithelial cells induced by FGF2 and FGF2 neutralizing. Kidney Int. 51: 301-309. 1997.
14. Yu D, Petermann A, Kunter U, Rong S, Shankland SJ, and Floege J. Urinary podocyte loss is a more specific marker of ongoing glomerular damage than proteinuria. J Am Soc Nephrol. 16: 1733-1741. 2005.

15. Breiteneder-Geleff S, Matsui K, Soleiman A, Meraner P, Poczewski H, Kalt R, Schaffner G, and Kerjaschki D. Podoplanin, novel 43-kd membrane protein of glomerular epithelial cells, is down-regulated in puromycin nephrosis. Am J Pathol. 151: 1141-1152. 1997.

16. Zou J, Yaoita E, Watanabe Y, Yoshida Y, Nameta M, Li H, $\mathrm{Qu} Z$, and Yamamoto T. Upregulation of nestin, vimentin, and desmin in rat podocytes in response to injury. Virchows Arch. 448: 485-492. 2006.

17. Yamanaka N and Shimizu A. Role of glomerular endothelial damage in progressive renal disease. Kidney Blood Press Res. 22: 13-20. 1999. 